\title{
Téoros
}

Revue de recherche en tourisme

\section{Pour un agenda de recherche sur le tourisme des gais}

\section{Philippe Connil}

Volume 19, numéro 2, été 2000

Le tourisme des gais et des lesbiennes

URI : https://id.erudit.org/iderudit/1071958ar

DOI : https://doi.org/10.7202/1071958ar

Aller au sommaire du numéro

Éditeur(s)

Université du Québec à Montréal

ISSN

0712-8657 (imprimé)

1923-2705 (numérique)

Découvrir la revue

Citer cet article

Connil, P. (2000). Pour un agenda de recherche sur le tourisme des gais. Téoros, 19(2), 11-15. https://doi.org/10.7202/1071958ar d'utilisation que vous pouvez consulter en ligne.

https://apropos.erudit.org/fr/usagers/politique-dutilisation/ 


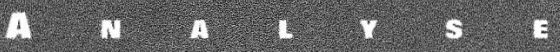

\section{Pour un agenda de recherche SUR LE TOURISME DES GAIS}

\section{Philippe Connil}

"Mais pourquoi une recherche sur le tourisme des gais ${ }^{1}$ ? se demandera le lecteur. La réponse est simple : dans les structures d'accueil et d'hébergement, il leur est difficile d'évoluer librement sans craindre une agression verbale, ou même physique, à caractère homophobe. L'élément sécurité est capital au tourisme des gais et lui confère une dimension socio-politique. Cet article, qui est le résultat des premiers pas de notre recherche doctorale, ne vise qu'à brosser, en prenant principalement à témoin l'expérience montréalaise, un tableau des pistes d'études potentielles et à proposer un agenda de recherche sur le tourisme des gais.

\section{MONTRÉAL : VILLE ET VILLAGE MODÈLES}

La ville de Montréal est devenue, à l'échelle mondiale, une ville modèle en matière de tourisme gai. Tourisme Montréal a mis en place une stratégie marketing pour accueillir les gais officiellement et sans aucune discrimination. Ces derniers sont sensibles au fait de lire une telle publication émanant d'un organisme officiel. Par ce cautionnement public, le lecteur, visiteur potentiel, éprouve ainsi un sentiment de sécurité et peut envisager avec sérénité son déplacement. À la suite d'un long processus d'appropriation de l'espace en milieu urbain, les gais et les lesbiennes en général, les associations et les entrepreneurs du Village en particulier ont créé un contexte favorable. Cependant une différence essentielle les sépare : l'action communautaire pour les associations et la démarche mercantile pour les professionnels. Le Village de Montréal revêt d'ailleurs aujourd'hui un caractère plutôt commercial, selon Frank W. Remiggi (Demczuk et Remiggi, 1998). Il est devenu un espace de consommation où l'esprit associatif semble avoir laissé peu à peu la place à des intérêts mercantiles. Divers/
Cité, organisateur émérite des journées de la Fierté gaie, qui a pour vocation première l'action communautaire auprès des gais et des lesbiennes ${ }^{2}$, travaille en étroite collaboration avec les instances du ministère de la Métropole et de Tourisme Montréal. L'opération rapporte quelques millions de dollars, à tel point que ces organismes ont commandé une enquête, en 1999 , pour mieux connaître le visiteur de ces journées de la fierté gaie et tenter de l'attirer en très grand nombre à d'autres occasions pendant l'année. Tourisme Montréal a rapproché l'offre du quartier gai et la demande de la clientèle étasunienne. Il réalise ainsi depuis quelques années, à partir de la Fierté gaie une vaste opération marketing.

\section{STONEWALL ET GAY PRIDE}

Ce Village est aussi le résultat d'une œuvre historique qui a pris racine après Stonewall, la plus grande révolte d'homosexuels du 28 juin 1969 à New York. La veille, dans la discothèque gaie new-yorkaise, Stonewall Inn, la police avait fait une descente qui avait tourné à l'émeute. Pendant les trois jours qui ont suivi, trois mille gais ont affronté les forces de l'or- dre. La manifestation de la Gay Pride est née en 1970 à New York pour commémorer le premier anniversaire des émeutes du Stonewall Inn. Depuis, chaque année à peu près à la même date, ont lieu les Gay and Lesbian Pride, les « marches de la fierté homosexuelle », de plus en plus nombreuses à travers le monde et de plus en plus importantes comme l'Europride en Europe et, pour la première fois en 1'an 2000, la World Pride, qui s'est déroulée à Rome début juillet.

Mais pourquoi tant de visibilité, la création de réseaux professionnels et l'émergence d'un tourisme gai institutionnalisé ? Didier Eribon (1999), évoque la vie de honte et de secret des homosexuels, des existences clivées, déchirées entre moi social et moi intime. L'un des enjeux majeurs de la visibilité est de montrer que l'homosexualité existe et d'interrompre ainsi le processus de reproduction de l'évidence hétéronormative. Dès la naissance, la masculinité se construit et s'affirme publiquement contre l'homosexualité dans toutes les situations sociales : à l'école et au travail, mais aussi dans le sport et dans toutes les structures récréatives, de loisirs et de tourisme. Ainsi, sortir de la honte pour un homosexuel est toujours perçu comme la proclamation de la fierté. Eribon aborde aussi le sujet de la «ghettoïsation»: le « ghetto » visible est d'abord et avant tout une manière d'échapper au « ghetto » invisible, au « ghetto » mental, auquel de nombreux individus sont contraints, car ils ne peuvent ou n'osent pas vivre leur homosexualité autrement que derrière l'écran de la dissimulation et de la honte. Il affirme également que ceux qui dénoncent la «ghettoïsation » expriment une réaction phobique à l'égard de la visibilité collective 
des gais, bien qu'en bout de ligne plusieurs gais souhaiteraient que leur visibilité ne soit pas confinée à un quartier de la ville...

Ce comportement de rejet s'observe face aux manifestations des gais et à leurs regroupements, d'autant plus dans leur tourisme où le phénomène de « ghettoïsation » existe bien, car la production gaie est cent pour cent gaie. Mais celle-ci ne s'adresse qu'à une des clientèles homosexuelles qui voyagent et, en l'occurrence, à une clientèle aisée, car les produits gais sont relativement onéreux. Alors, où vont les autres gais? Ils ont le choix entre la clandestinité ou encore rejoindre les grandes métropoles urbaines où ils seront assurés de trouver le «Village» de la reconnaissance. Pour plusieurs gais, bien entendu, l'orientation sexuelle n'influence pas le choix de la destination, particulièrement si ils voyagent en couple.

\section{LE VILLAGE DE MONTRÉAL : PÔle TOURISTIQUE INTERNATIONAL}

Le quartier gai dans la ville reflète l'espace urbain de liberté des homosexuels. Ceux-ci y retrouvent une identité et un certain nombre de marquages qui leur assurent une évolution spatiale plus naturelle, plus sereine et plus sécurisée par rapport aux autres territoires. Le Village est pour beaucoup un lieu de passage et de consommation. Le travail réalisé par les services de la recherche et du développement de Tourisme Montréal (1999) souligne que les gais font partie des nouvelles clientèles touristiques urbaines qui voyagent plus aujourd'hui par identité et centre d'intérêt commun que par choix initial de destination géographique.

Les résultats de son étude sur Le tourisme gai confirment l'enjeu de cette stratégie le nombre de gais et de lesbiennes aux États-Unis s'élève à 15 millions de personnes, $64 \%$ des touristes gais ont des revenus de 40000 dollars américains et plus et $91 \%$ possèdent un diplôme d'études collégiales ou universitaires. Les hommes gais ont deux fois plus tendance à voyager que le reste de la population américaine. Les choix touristiques révèlent que les gais et les lesbiennes visitent d'abord une destination où ils pourront se sentir à l'aise et réputée gay friendly, où ils seront bien accueillis. Beaucoup préfèrent loger près d'un quartier ou d'une communauté gaie

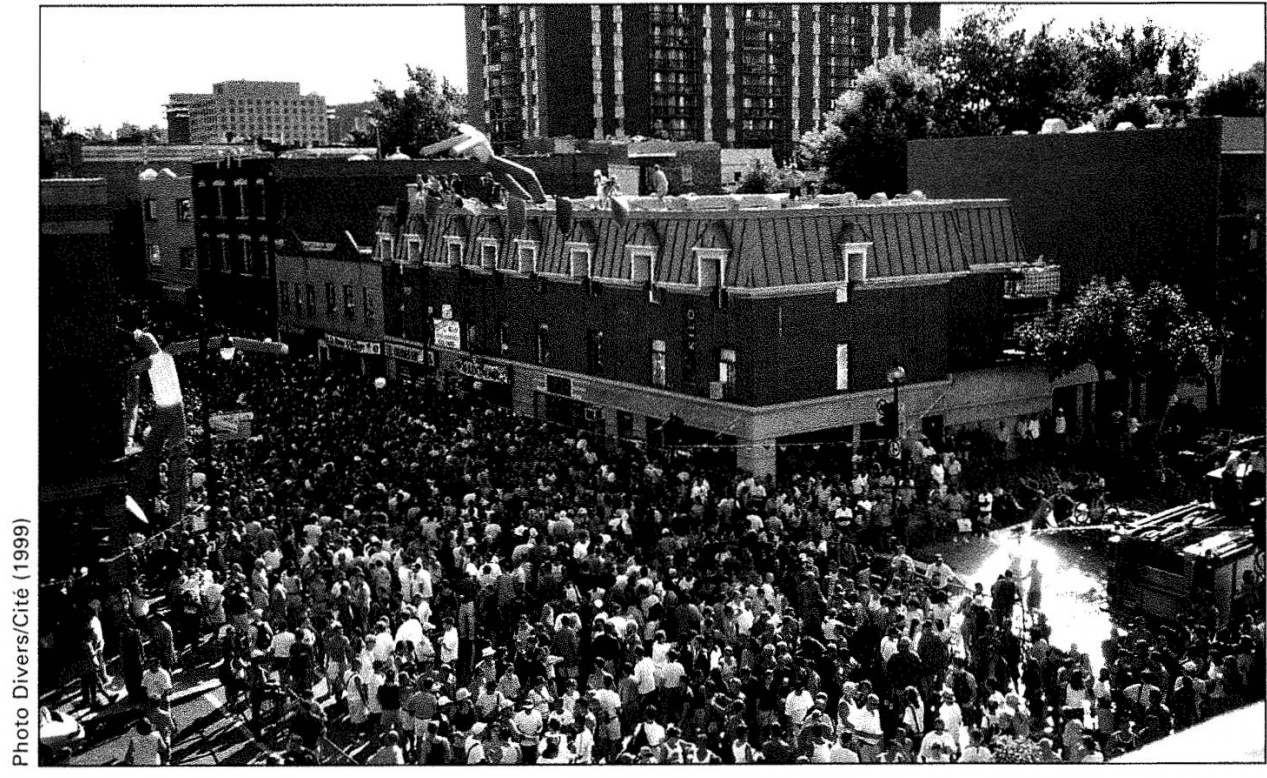

Les participants et les spectateurs se retrouvent pour faire la fête dans le Village gai montréalais après le défilé de la Fierté.

pendant leur voyage. Les gais ont plutôt tendance à visiter de grands centres urbains et $93 \%$ d'entre eux recherchent des tour opérateurs et des fournisseurs gais. Ils sont très loyaux aux entreprises qui démontrent de l'intérêt pour leur communauté. Montréal, toujours d'après l'étude, est perçue comme une destination gay friendly et considérée comme l'une des villes les plus tolérantes au monde envers les gais ${ }^{3}$. Cette tolérance se manifeste aussi par la commercialisation sexuelle présente dans le Village mais aussi sur le Plateau MontRoyal et même en banlieue, ou les bars de danseurs nus et les saunas se multiplient. Avec ses différents événements et festivals, elle est de plus en plus reconnue sur la scène gaie internationale : le Black and Blue et les autres rave parties bénéfices, mais aussi les événements sportifs gais et enfin, des congrès exceptionnels comme celui de l'International Gay \& Lesbian Travel Association (IGLTA) en mai 1998 et celui de la Fédération des Jeux Gais en avril 1999. Les touristes gais viennent profiter de l'atmosphère de réjouissance de son Village et les Américains y sont nombreux lors des longues fins de semaine.

En quelques années, le Village de Montréal est donc devenu un pôle touristique de premier plan par la concordance de deux actions : celle des entrepreneurs du Village qui ont développé leurs structures commerciales et celle de Tourisme Montréal qui a mis en place une stratégie marketing. Ce dernier utilise toutes les ressources - 10gistiques, financières et humaines - du
Village pour faire de la Ville une destination gaie locale et internationale. Les enjeux sont devenus internationaux grâce à de vastes réseaux de promotion de destinations qui génèrent des flux touristiques, des dépenses et des retombées considérables pour les villes d'accueil. Le Village de Montréal offre les mêmes marquages que ceux de New York, Boston, Paris, Londres ou Sydney : ils déclenchent donc entre eux des déplacements d'une population gaie qui s'approprie l'espace, le modifie et le façonne par une nouvelle démarche à la fois commerciale et touristique.

Frank Mort (1998) présente sa recherche sur les relations entre le commerce et le façonnement de l'identité gaie dans des quartiers spécifiques. Il a déjà démontré dans des écrits antérieurs que ces différents territoires représentent des scènes qui favorisent les relations humaines et sociales. Il propose de poursuivre son investigation et de soumettre l'idée qu'espace et identité forment un couple complet, allant même jusqu'à employer le terme distique. Il prend l'exemple du quartier londonien de Soho qui est devenu dès 1950 le théâtre de la consommation gaie. Cinquante ans plus tard, ce territoire continue d'être l'expression de la communauté gaie et son espace de consommation. Frank Mort cite d'autres quartiers similaires : Oxford Street et Darlinghurst à Sydney, le Marais et la Bastille à Paris, El Bario Chueca à Madrid, que les guides touristiques gais mondiaux mentionnent d'ailleurs. Tous ces centres 
font la promotion de leur histoire, de leur culture et de leur version contemporaine de la bohème. Leur population consomme de façon semblable, de façon rituelle selon Frank Mort, quel que soit le pays, et les membres de ces communautés se reconnaissent facilement. Cette consommation globale représente un segment de marché particulier et ses consommateurs partagent des styles de vie similaires dans des pays fort éloignés les uns des autres. Ils voyagent fréquemment entre ces lieux de l'avant-garde de la mode, du design, de la musique et de l'architecture.

Les pays d'Europe, d'Amérique du Nord et l'Australie emboîtent aujourd'hui le pas et suivent l'exemple institutionnel de Montréal, non seulement individuellement, mais aussi à travers de vastes réseaux de promotion : à l'automne 1999, les villes de Manchester, de Munich, de Sydney, de Paris et de Montréal se sont associées pour promouvoir leur destination auprès des gais et des lesbiennes de six métropoles étasuniennes : Dallas, Los Angeles, San Francisco, New York, Fort Lauderdale et Boston. Les représentants à Los Angeles de la Maison de la France, placée sous la tutelle du ministère du Tourisme français, ont à cette occasion présenté la première brochure Gay Friendly France pour vendre la destination aux États-Unis.

\section{LES JEUX GAIS}

Soucieuses de montrer un visage homophile et de prouver un accueil ouvert et respectueux envers les gais et les lesbiennes du monde entier, les villes sont par ailleurs souvent candidates aux Gay Games. Les Jeux, créés par l'Américain Tom Waddell, médecin spécialiste des maladies infectieuses et décathlonien aux Jeux Olympiques de Mexico en 1968, ont été organisés pour la première fois à San Francisco en 1982. Ils devaient rompre avec l'image stéréotypée de l'homosexuel efféminé et permettre à la communauté gaie de s'affirmer fièrement et en toute visibilité. Pierre Bourque, maire de Montréal, était allé à Denver avec les représentants de la Chambre de commerce gaie du Québec pour soutenir la candidature de sa Ville aux prochains Gay Games de 2002. Le dossier, pourtant très complet et bien préparé, n'a pas retenu l'attention du jury qui a préféré Sydney. À New York, en 1992, les retombées économiques des Gay Games ont été évaluées à 110 millions de dollars. Montréal est à nouveau candidate pour les Gay Games de 2006, mais la manifestation a atteint une telle importance que l'organisation est maintenant affaire de spécialistes. Montréal est, à ce titre, bien placée, car elle possède l'expérience logistique et la gestion financière de plusieurs manifestations gaies. Mais, elle se trouve en concurrence avec d'autres grandes métropoles comme Berlin, Paris, Chicago et la Nouvelle-Orléans.

\section{LES DESTINATIONS GAIES}

En Europe, l'Espagne est certainement le pays qui accueille le plus grand nombre de gais pendant la saison estivale. Les principaux sites sont: Sitges, station balnéaire à cinquante kilomètres au sud de Barcelone, Benidorm, au nord d'Alicante, Torremolinos, près de Malaga au sud de la péninsule et, enfin, Ibiza aux Baléares et l'île de la Grande Canarie qui reçoivent plus d'Allemands que de Français. Ces destinations offrent les avantages que les gais recherchent pour leurs vacances d'été : la mer, le soleil, la plage et une animation nocturne intense.

Pendant l'hiver, les gais profitent aussi des longs week-ends pour visiter les grandes capitales européennes, en particulier Londres. La ville la plus vaste d'Europe abrite une grande communauté homosexuelle et assure à ses visiteurs de nombreuses découvertes. Le tunnel sous la Manche facilite désormais les relations entre l'Angleterre et la France et le Train à Grande Vitesse Eurostar met Londres à trois heures de Paris. Amsterdam aussi est devenue facilement accessible par TGV. Les gais, qui représentent $25 \%$ de la population, évoluent dans différents quartiers du centre ville, dits des canaux, dans les rues Kerkstraat, Reguliersdwarsstraat et Warmoesstraat. Dans ce tour d'horizon touristique européen non exhaustif, trois autres grandes capitales sont très en demande pour de courts séjours : Berlin, Madrid et Barcelone.

De novembre à mars, alors que 1'hiver s'installe en Europe, l'Australie reste une destination de choix très prisée des gais, ceux du moins qui disposent d'un bon budget de vacances. À quelque 17000 kilomètres de Paris, la destination fait rêver et à juste titre : de Cairns à Melbourne, en profitant de la barrière de corail et en visitant la Gold Coast, sans oublier au sud

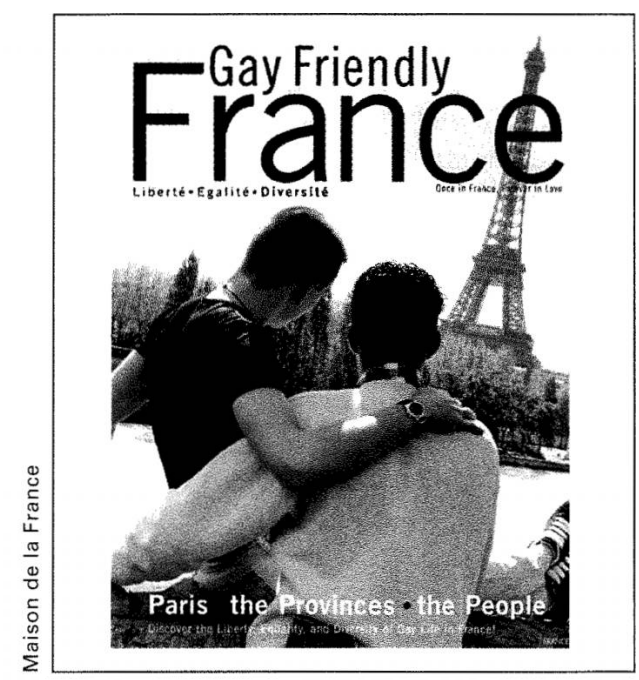

La nouvelle brochure de la Maison de la France pour le marché gai étasunien.

les vignobles de Yarra Valley et au nord la région subtropicale de la péninsule de Cap York. Sydney offre tous les services culturels, sportifs et ludiques que consomment les gais : elle est devenue la destination vedette long-courrier par son célèbre Gay and Lesbian Mardi Gras. La manifestation attire plus de 500000 personnes et le jour de la grande parade, le centre de la ville tout entier est fermé à la circulation pour faciliter le défilé.

Les États-Unis sont beaucoup plus abordables financièrement au départ de l'Europe. Les deux métropoles les plus cotées par les gais sont New York et San Francisco avec leurs quartiers gais : Greenwich Village et Castro. La Nouvelle-Orléans organise trois manifestations où de nombreux gais se retrouvent pour faire la fête et se déguiser: Mardi Gras en février, Southern Decadence début septembre et Halloween fin octobre. Le célèbre French Quarter et en particulier Bourbon Street accueillent une population gaie - tant du Middle West que de nombreux français attirée par le caractère francophone de la ville et son climat très agréable toute l'année. Les vacances en Amérique peuvent connaître un épisode romantique à Key West, dans l'archipel de l'extrême sud de la Floride, à Miami, à Fort Lauderdale et sur la presqu'île de Cap Cod, dans l'état du Massachusetts, au large de Boston, où le tourisme de Provincetown est fortement orienté vers les gais. Au Canada, en dehors de la province de Québec où la réputation de la destination n'est plus à faire, les grandes villes d'Ottawa, de Toronto et de 
Vancouver possèdent chacune leur quartier gai. Elles organisent aussi leur Gay Pride et réservent un bon accueil aux voyageurs homosexuels, mais regardent avec quelque peu d'admiration, et sans doute de jalousie, les performances de Montréal en matière de tourisme gai.

En Amérique centrale, le Costa Rica semble le pays le plus ouvert au sujet et plusieurs établissements hôteliers de la station balnéaire de Quepos sont représentés par des tour opérateurs gais. En Amérique du Sud, Rio de Janeiro et São Paulo au Brésil se concurrencent au moment du carnaval. En Argentine, Buenos Aires a organisé sa Gay Pride en novembre dernier, car les saisons sont inversées par rapport à l'hémisphère nord. Cependant, dans ces pays du sud, il est encore parfois difficile pour deux hommes ou deux femmes qui voyagent ensemble d'obtenir une chambre double avec un grand lit... Ce tour du monde serait incomplet sans préciser que 1'Afrique du Sud et la ville du Cap développent officiellement, depuis deux ans maintenant, une politique touristique ouvertement gay friendly.

Retour en Europe et en France où l'organisation des professionnels du tourisme gai était jusqu'à présent plutôt axée sur l'émission de clientèle vers l'ensemble des destinations citées précédemment. Depuis un an, le ministère du Tourisme vend la destination Gay Friendly France à l'étranger et plusieurs agences réceptives privées, à Paris et sur la côte d'Azur, ont été créées pour répondre à la demande spécifique des voyageurs homosexuels.

\section{AGENDA \\ DE RECHERCHE SUR LE TOURISME DES GAIS}

Encore embryonnaire, la recherche visant à mieux comprendre le tourisme pratiqué par les gais pourrait ainsi se présenter sous quatre volets :

\section{1- L'appropriation de l'espace urbain} par les gais avant et après Stonewall. Des auteurs récents donnent une autre dimension aux études gaies et lesbiennes, qui s'étaient jusqu'à présent limitées au mouvement de libération de l'après Stonewall. Dans son dernier ouvrage, George Chauncey (1994) fait revivre la vie gaie new-yorkaise de la fin du $\mathrm{XIX}^{\mathrm{e}}$ siècle et du début du XX $\mathrm{XX}^{\mathrm{e}}$. Son analyse historique contribue largement au savoir. Elle porte son livre en

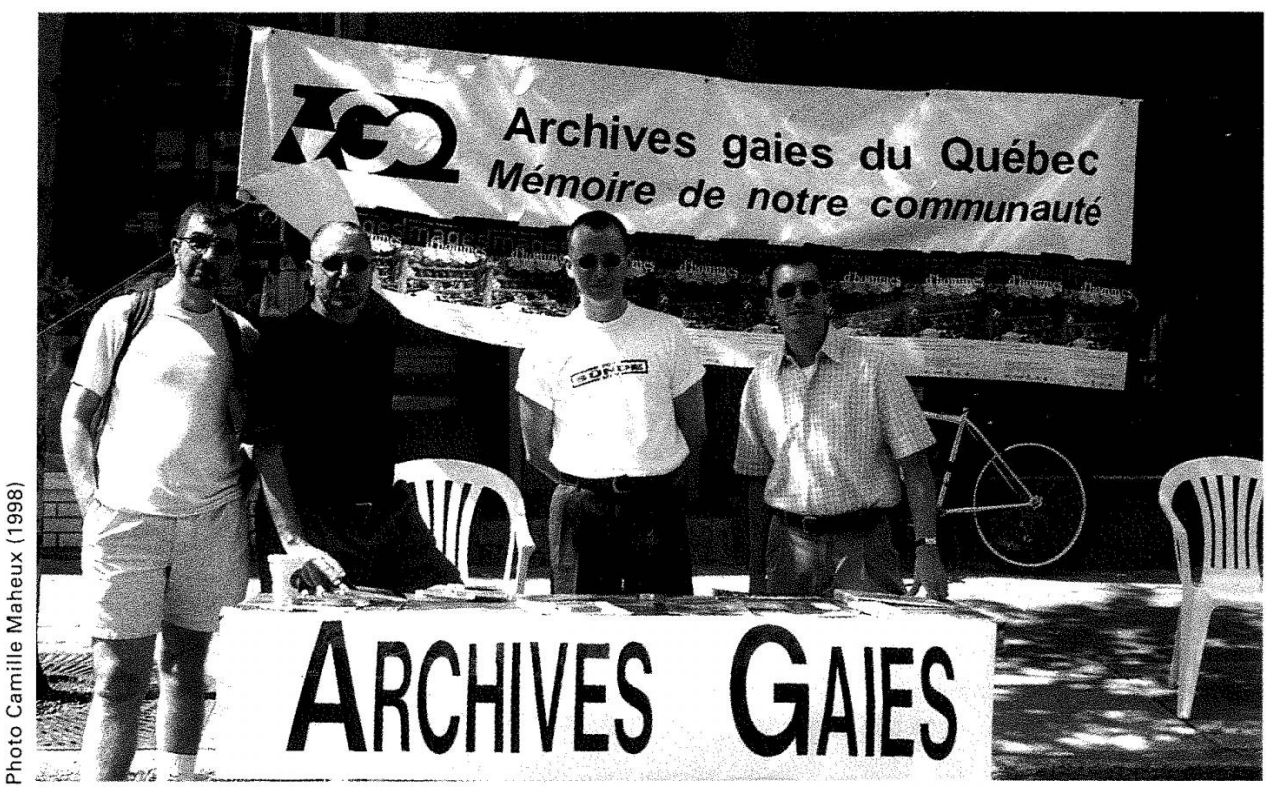

Les Archives gaies du Québec, incontournable source d'informations textuelles et visuelles, lors de la journée communautaire des fêtes montréalaises de la Fierté.

référence de la sous-culture gaie d'avant-guerre, qui était concentrée dans les quartiers de classe ouvrière, donc en zone péri-urbaine. Ces nouvelles études, selon Hood, (1998 : 782792), s'inscrivent directement dans l'histoire urbaine par :

- la présence des homosexuels dans les grandes villes américaines au-delà du siècle dernier,

- la lumière qu' apportent les études sur une dimension négligée de l'expérience urbaine en matière d'organisation de la sexualité,

- et la compréhension qu'elles donnent à la dimension sociale de l'espace urbain et à la composition de la communauté.

2- L'étude de ce segment de clientèle. Les gais veulent aujourd'hui vivre leur sexualité sans se cacher et consommer dans un espace identitaire. Ils souhaitent être reconnus à la fois par la société et par les entreprises dans leurs comportements d'achat. Dans cette communauté, où les revenus annuels sont en général sans charge de famille ni d'éducation d'enfants, la part du budget consacrée aux loisirs est tout naturellement supérieure aux autres situations sociales traditionnelles. Les gais ont la possibilité de sortir et de dépenser plus souvent. Ils sont en attente de structures commerciales plus nombreuses, notamment dans l'élaboration de formules de vacances, avec un pouvoir d'achat sur ces produits qui intéresse les professionnels du secteur.

3- La variable marketing des entrepreneurs locaux et des grands acteurs institutionnels. Les gais disposent d'une plus grande liberté de mouvement, de voyage et d'action, avec une capacité de dépense et de décision plus importante et plus rapide que la clientèle familiale. Ils représentent des groupes socio-spaciaux que les professionnels du tourisme ne peuvent ignorer dans la réflexion de leur stratégie marketing. L'organisation spatiale, pour répondre à la demande identitaire des gais, à leur consommation et à leur sexualité, fait intervenir de nombreux acteurs municipaux, au-delà des décideurs touristiques.

4- Le jeu de l'appropriation de l'espace urbain et de l'insertion sociale de la communauté gaie dans la ville par le biais de ses déplacements touristiques. En trente ans, les gais ont approprié leur territoire et, cette fois, de façon visible. Les Villages du monde entier sont devenus des émetteurs et des récepteurs de clientèles touristiques. Ces visiteurs, ces touristes et ces excursionnistes, transportent leur culture et leur exotisme. Le tourisme concourt à l'épanouissement individuel et collectif des gais. Ses activités tendent à exporter les droits, les comportements et les coutumes dans les espaces urbains d'accueil, jusqu'à en modifier le paysage. 


\section{LA NÉCESSAIRE PLURIDISCIPLINARITÉ}

Pour conclure, notons que notre recherche sur le tourisme des gais s'inscrit ainsi dans le corpus des études urbaines. Les études gaies et lesbiennes, à notre sens, ne devraient pas être isolées dans un programme spécifique, mais bien au contraire trouver espace et reconnaissance au sein de chaque domaine d'étude et avoir droit de cité dans les différents départements des facultés des sciences sociales. Les différentes disciplines, qu'il s'agisse d'histoire, de géographie, d'économie, de sciences politiques, d'études littéraires, etc., ont toutes été façonnées et trouvent toutes écho dans la communauté gaie et lesbienne autant qu'hétérosexuelle. Le cloisonnement des savoirs comporte un risque bien réel de perdre contact avec les autres secteurs de la vie et du travail intellectuels (Eribon, 1998). Cependant, il faut développer, dans un premier temps, un champ de recherche exclusif qui peu à peu s'intégrera dans un ensemble de réflexions générales sur la ville.

Détenteur d'un DESS en Aménagement et gestion des stations touristiques de l'Université de Bordeaux 3 (France), Philippe Connil est présentement au doctorat en Études urbaines à l'Université du Québec à Montréal (Canada). Son doctorat porte sur le développement et les manifestations actuelles du tourisme pratiqué par les gais et les lesbiennes en prenant comme assises le cas de la ville de Montréal.

Un comité de lecture a lu et accepté ce texte

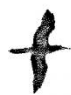

\section{Camping pour hommes seulement}

- Section standard et naturiste

- 300 sites boisés pour tentes et roulottes

- Piscine, terrasse, bars, discothèque, restaurant

- Terrains sportifs

- Location de roulottes et de chambrettes

\section{Camping Plein Bois}

550 Chemin St-Henri

Ste-Marthe (Vaudreuil) JOP 1 WO

\section{Tél.: 450-459-4646,} $1-888-459-4646$

fax.: $450-459-9417$

Site web:

www.campingpb.qc.ca

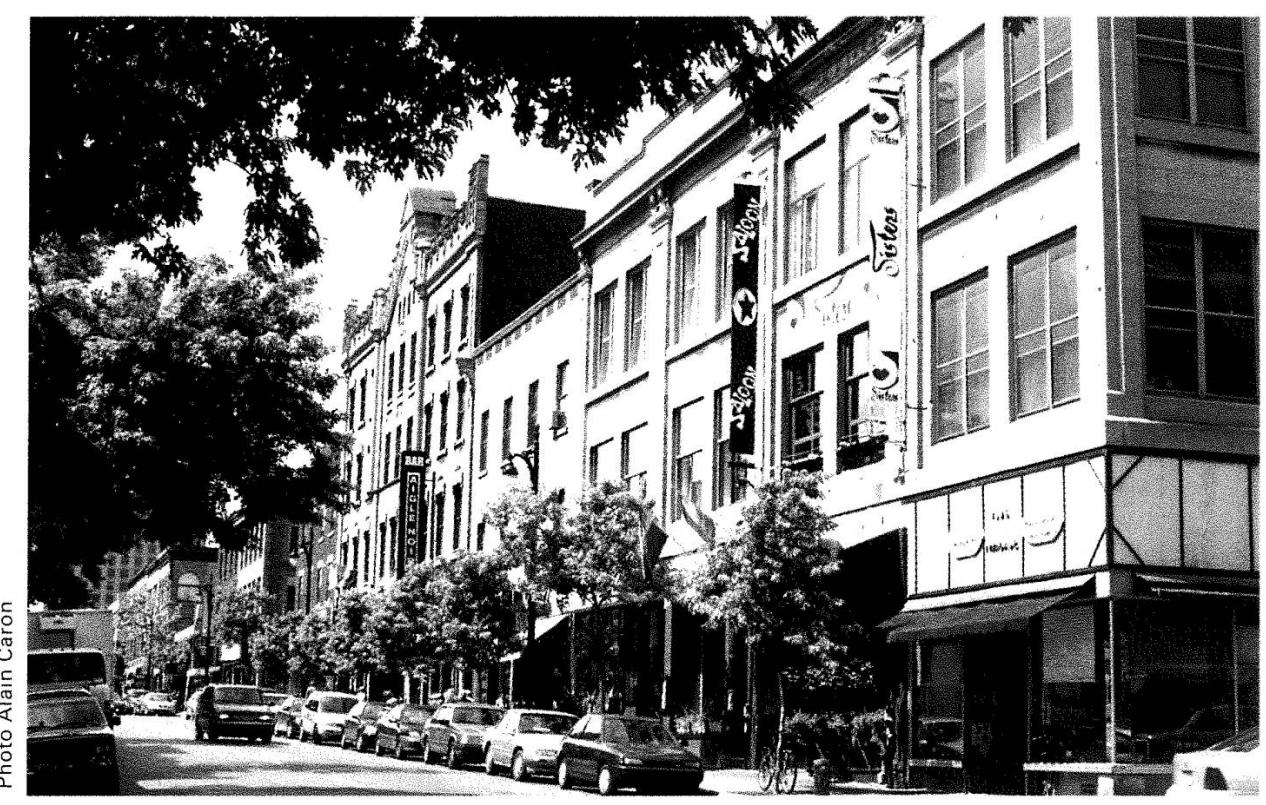

La rue Sainte-Catherine Est, artère commerciale du Village gai montréalais.

\section{NOTES}

1 Par le mot « gais », l'auteur du présent article entend parler des homosexuels hommes et femmes. Cependant, leurs réalités économiques, sociologiques et politiques diffèrent, ce qui a des répercussions sur les tourismes qu'ils pratiquent. Les attentes et les comportements des gais et des lesbiennes sont donc différents, bien que cet article soit de portée générale. Il convient aussi de distinguer le tourisme gai qui constitue une offre touristique destinée au gais du tourisme des gais qui couvre toutes les formes de tourisme pratiquées par les gais, dont certaines ne sont pas influencées par l'orientation sexuelle.

2 Au delà des superlatifs (un total de 950000 spectateurs sur place et devant leur téléviseur en 1999) et des reconnaissances (prix Ulysse 2000 de Tourisme Montréal dans la catégorie «manifestations touristiques avec un budget de moins d'un million de dollars»), la directrice de Divers / Cité Suzanne Girard insiste avant tout sur les effets fédérateurs pour les organismes communautaires montréalais et sur la visibilité de la communauté. Un élément touristique stratégique : le défilé de la Fierté montréalais a lieu au début du mois d'août, contrairement à tous les autres placés à la fin juin pour commémorer Stonewall... Les organisations collaborent au sein d'un réseau mondial appelé InterPride qui regroupe 125 villes.

3 De par sa culture latine dans un continent anglo-saxon, la métropole québécoise est, au dire de plusieurs, plus « libertine » que les autres métropoles nord-américaines. Son caractère multiethnique et sa stabilité sociale favorisent aussi cette ouverture.

\section{BIBLIOGRAPHIE}

Amnistie Internationale (1997), Breaking the Silence. Human Rights Violations Based on Sexual Orientation, Londres, Amnistie internationale United Kingdom, traduction française (1998) Briser le silence. Violations des droits de l'Homme liées à l'orientation sexuelle, Paris. Amnistie internationale.

Chauncey, George (1944), Gay New York: Gender, Urban Culture, and the Making of the Gay Male World, 1890-1940, New York, Basic Books.

Demczuk, Irène, et Frank W. Remiggi (1998), Sortir de l'ombre. Histoires des communautés lesbienne et gaie de Montréal, Montréal, VLB Éditeur.

Eribon, Didier (1999), Réflexions sur la question gay, Paris, Fayard, 1999.

Eribon, Didier (sous la direction de) (1998), Les études gay et lesbiennes, Colloque du Centre Georges Pompidou, 23 et 27 juin 1997, Paris, Éditions du Centre Pompidou.

Hood, Clifton (1998), « New Studies in Gay and Lesbian History », Journal of Urban History, 24 (6), Septembre.

Mort, Frank (1998), « Cityscapes: Consumption, Masculinities and the Mapping of London Since 1950 », Urban Studies, Vol. 35, nos 5 et 6, p. 889-907.

Tourisme Montréal (1999), Le tourisme gai, Montréal. 